\title{
Demonstrating of a Standing Wave - Thermoacoustic Refrigerator
}

\author{
Ali Alahmer ${ }^{a}$, Mohammed Omar $^{b, c}$, Mohammad Al Zubi ${ }^{a}$ \\ ${ }^{a}$ Department of Mechanical Engineering, Tafila Technical University, Tafila, Jordan, P. O. Box 179, 66110 \\ ${ }^{b}$ Automotive Engineering Department, Clemson University, International Center for Automotive Research CU-ICAR, \\ Greenville, SC 29607, USA \\ ${ }^{c}$ Department of Engineering Systems \& Management, Masdar Institute of Science \& Technology, Abu Dhabi, UAE
}

\begin{abstract}
This manuscript begins with a full description of Thermoacoustic Refrigerator (TAR) technology followed an analysis of the elements necessary for successfully designing the various components of a TAR. The authors developed and tested a standing wave thermoacoustic refrigerator (TAR) to study the effects of stack plate thickness, load and stack temperature variation on TAR performance. They then used a CFD simulation to simulate the performance of the TAR, particularly the temperature and velocity within.
\end{abstract}

Keywords: Thermoacoustic, Refrigerator, Standing wave, Stack

\section{Introduction}

As the concept of thermoacoustics remains mostly unknown, developing methods to construct and develop thermoacoustic refrigeration systems does as well. There is a positive trend in the amount of published papers about the subject, however, which describes the use of cryogenics, commercial refrigeration or electricity generation [1-3]. Thermoacoustic science is defined as a field of study that deals with the conversion of sound energy into heat and vice versa, in which sound energy is used to transfer the heat is transferred from a cold to warm medium [4]. Thermoacoustic device are categorized as either standing-wave devices, which are described with the Brayton cycle; or travelling-wave devices, which are described with the Stirling thermodynamic cycle [5]. Thermoacoustic refrigerators are superior to the vapor compression refrigerator in that as they need air or inert gas, environmentally hazardous refrigerants are not necessary. Also the lack of moving components in thermoacoustic refrigerators makes the devices simple and reliable, which in turn results in low fabrication costs. Though these systems are superior in several facets, they unfortunately lack the thermal efficiency of conventional refrigeration technologies [3, 6-7].

There has been little research involving the numeric optimization and design necessary for constructing standing wave thermoacoustic refrigerator, however [2, 8-11]. Here we briefly discuss the processes, properties and limits involved in designing and manufacturing the components of these refrigerators, with specific emphasis upon the key design inherent in such thermoacoustic refrigeration design. We begin, in section two, with a discussion of the basic operation of a thermoacoustic device and its controlling factors. In section three, we present our research methodology and specify the components necessary for a successful thermoacoustic design in section four. In section five, we describe the various measurements that affect thermoacoustic performance (e.g. the effect of varying the stack temperature), and summarize our findings through conclusion in section six.

\section{Principles of Thermoacoustic Refrigerator Technology}

The configuration of a standing wave thermoacoustic refrigerator, as depicted in Figure 1, consists of six main parts: an acoustic driver within a housing, an acoustic resonator (tube) filled with the working fluid, a stack of thin parallel plates installed inside the resonator-known as a regenerator in travelling-wave devices; and two heat exchangers (hot and cold) attached to both ends of the stack $[2,4]$. This refrigerator uses a driver to create sound waves, which are used to cool the machine. We next describe in detail the design and construction of the different components.

* Corresponding author.

E-mail: a.alahmer@ttu.edu.jo

(c) 2013 International Association for Sharing Knowledge and Sustainability

DOI: $10.5383 /$ ijtee.06.02.005 


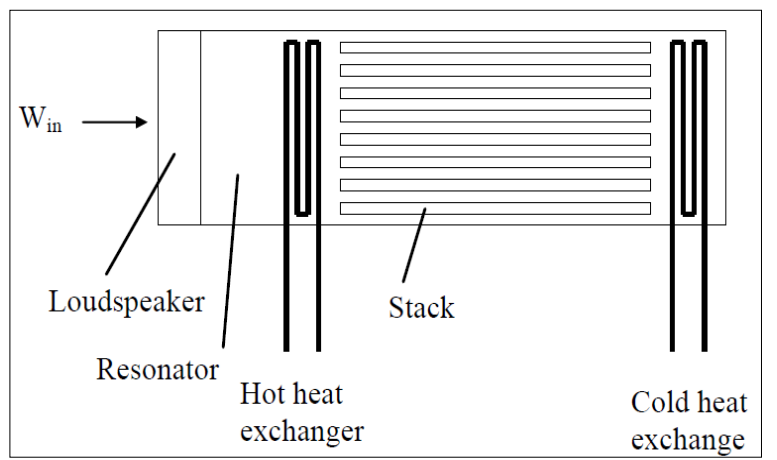

Fig. 1. Standing wave thermoacoustic refrigerator demonstration.

\subsection{General Theory}

The longitudinal standing sound wave causes the gas particles to oscillate in parallel to the walls of the stack. The alternating compression and rarefaction of the gas causes the local temperature of the gas to oscillate due to the adiabatic nature of sound waves [12]. Figure 2 shows the trace of the four stages of the thermoacoustic refrigeration cycle, particularly as regarding the circulation of the refrigerant fluid. In the first stage, known as the compression stage, the fluid is compressed as it moves to the left from a lower pressure region to a higher pressure region, which causes an increase in its temperature. In the subsequent heat rejection stage, the temperature of the fluid parcel in this compressed state, which is greater than the local stack temperature, causes the gas to eject the heat back into the stack, and cool the fluid. During the third stage, also known as the expansion stage, the pressure of the fluid parcel is reduced, which in turn causes it to expand and decrease in temperature. In the fourth and final stage, known as the heat withdrawal stage, the fluid parcel temperature is less than the local stack temperature, causing heat transfer from the stack to the fluid. It is clear that in this cycle, sustainability relies upon the (a) thermal delays associated between the stack and the working fluid, and (b) resonant acoustic environment in which the cycle occurs.

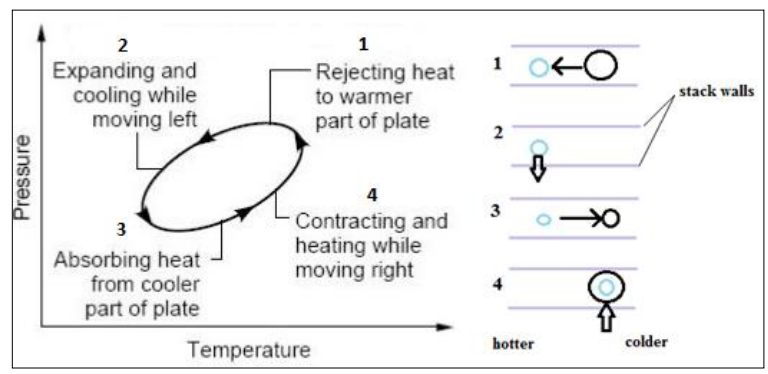

Fig. 2. Represent the thermoacoustic refrigerator cycle.

\subsection{Factors Affecting on the Performance of Thermoacoustic Refrigerator}

The first most important factor in the component design of a thermoacoustic refrigerator that directly affects the performance of a thermoacoustic refrigerator is the thermal penetration depth. This depth, which is approximately the distance that heat can diffuse through the gas within a time constant, is expressed as $1 / \pi f$ $\delta_{\mathrm{k}}=\sqrt{\frac{2 \mathrm{k}}{\mathrm{P}_{\mathrm{m}} \mathrm{C}_{\mathrm{p}} \omega}}=\sqrt{\frac{\mathrm{k}}{\mathrm{P}_{\mathrm{m}} \mathrm{C}_{\mathrm{p}} \pi f}}$

where $\mathrm{f}$ is the frequency of the standing wave, $\mathrm{k}$ is the thermal conductivity of the working fluid, $p_{m}$ is the mean fluid density, $\mathrm{C}_{\mathrm{p}}$ is the isobaric specific heat per unit mass, and $\omega$ is the radial frequency. The physical meaning of thermal penetration depth is a measurement of how well a fluid can transfer heat through its boundary. A large thermal penetration depth permits a greater heat transfer between the stack walls and the gas, increasing the overall efficiency of the thermoacoustic refrigerator [6]. In standing-wave thermoacoustic devices, a key geometrical requirement is that the smallest transverse dimension in the stack be roughly 1 to 3 times the penetration depth [13].

The second factor is viscous penetration depth which is approximately the distance over which the momentum can diffuse in a time $1 / \pi f$

$\delta_{v}=\sqrt{\frac{2 \mu}{\rho_{\mathrm{m}} \omega}}=\sqrt{\frac{\mu}{\rho_{\mathrm{m}} \pi f}}=\sqrt{\frac{v}{\pi f}}$

where $\mu$ is the dynamic viscosity, and $v$ is the kinematic viscosity. Thus, within $\delta_{v}$ of solid surfaces, viscous shear causes gradients in both the oscillatory velocity and the displacement. A fluid's viscous penetration depth can be viewed as a measure of the frictional losses within the fluid. A small viscous penetration depth indicates that the losses per unit area due to the viscous effects will be lower, which is important in the many small pores of the stack where the surface area is large $[6,13]$. The relationship of the thermal and viscous penetration depths are indicated by a fluid's Prandtl number, defined as the ratio between the viscous and thermal penetration depth, which is expressed as

$\sigma=\left(\frac{\delta_{v}}{\delta_{\mathrm{k}}}\right)^{2}=\frac{\mathrm{C}_{\mathrm{p}} \mu}{\mathrm{k}}$

Therefore, a lower Prandtl number is desirable as it indicates that the gains with respect to thermal considerations will outweigh the viscous losses $[6,13]$.

The third factor is the critical longitudinal temperature gradient $\nabla \mathrm{T}_{\text {crit }}$, which is defined as the temperature at which no heat will be transferred through the stack.

$\nabla \mathrm{T}_{\text {crit }}=\frac{\mathrm{p}}{\xi \rho \mathrm{cp}}$

Here, heat is transferred between the hot and cold ends of the stack from the lower temperature to the higher temperature causing refrigeration, where $\mathrm{p}$ and $\xi$ are the acoustic pressure and displacement amplitudes of the sound wave, respectively. The next factor is the drive ratio DR, which is defined as the acoustic pressure amplitude, P1 divided by the mean pressure, $\mathrm{Pm}$.

$\mathrm{DR}=\frac{\left\|\mathrm{P}_{1}\right\|}{\mathrm{P}_{\mathrm{m}}}$ 
The DR is a useful indicator of the nonlinearity of the system, since it is linked to the acoustic Mach number and hence the flow velocity. This ratio should be kept sufficiently low so as to avoid acoustic nonlinearities such as turbulence [14].

The last factor is the blockage ratio, BR, which is defined as the fraction of the cross sectional area of the resonator unblocked for the gas movement by the stack plates [3-4].

$$
\mathrm{BR}=\frac{\text { Ratio of open aria }}{\text { Total cross sectional aria }}=\frac{\mathrm{A}_{\mathrm{u}}}{\mathrm{A}_{\text {tot }}}=\frac{\mathrm{y}_{0}}{\mathrm{y}_{0}+l_{0}}
$$

where $A_{\mathrm{u}}$ is the unblocked cross sectional area, $A_{\text {tot }}$ is the total cross sectional area, $Y_{0}$ is half of the gap between the stack layers and $l_{0}$ is half of the thickness of the stack layers. Often used to describe the portion of open area through a stack or regenerator, the purpose of the BR is to characterize the system performance in terms of various stack configurations [9].

\section{Design Strategy}

Many parameters must be considered when designing a standing-wave TAR (e.g. the stack length and position, the resonator dimensions, the working gas properties, the driver parameters, and the operating conditions). When initiating the design, the number of variables must first be reduced. The first step in this reduction entails selecting a working gas because all components depend on the physical properties of a fluid. Next, the operating conditions must be selected so the average operating pressure and the mean temperature due are more dependent upon the other parameters, which may be easily adjusted as needed. After determining the initial choices, we then begin to design the components, starting with the stack. In that the stack is often made of a material that is both expensive and difficult to machine, it may be difficult to construct a stack to meet predetermined specifications. After selecting a few of the stack parameters, we may design our resonator accordingly, and select a driver from there are, of course, some situations in which a different design strategy may be better, but this method was deemed appropriate due to the availability of certain resources (i.e. helium and a porous plastic material). After successfully constructing the TAR, we began our experiments by setting the frequency at a reasonable value of $350 \mathrm{~Hz}$ using a function generator adjusted by the power amplifier. We next switched on the speaker, and recorded the temperatures at the hot heat and cold heat exchangers using thermocouples at 5 minute intervals. The duration of this experiment was approximately 90 minutes.

\section{Thermoacoustic Component Design}

\subsection{Working Gas}

The working gas must have a large thermal penetration depth, $\delta_{\mathrm{k}}$, and a small viscous penetration depth, $\delta v[6,13]$. The inert gas used in our experiments was helium, as it contains the greatest sound velocity and thermal conductivity of all inert gases [13, 14], making it an excellent design choice. Also, the high sound velocity and high thermal conductivity and the associated increased thermal penetration depth $\delta_{k}$ allows the construction of relatively high-frequency devices without being too small in dimension. This high thermal conductivity in turn increases the thermal penetration depth of the device, which increases the stack geometry to sizes that can be accommodated by relatively inexpensive manufacturing methods [3]. All helium properties are tabulated in Table 1.

\subsection{Stack Design}

The stack is positioned within the resonator such that it is between a pressure antinode and a velocity antinode in the sound wave. The main purpose of the stack is to provide thermal capacity and maintain imperfect thermal contact with the oscillating fluid. This imperfect thermal contact between the gas and the solid stack will, in turn, lead to a phase shift between the temperature and pressure oscillations of each gas particle and convert the acoustic pressure oscillations into a temperature gradient. Another purpose of the stack is to thermally isolate the gas particles from the outside environment to allow the adiabatic transport of heat from one heat exchanger to another [15]. At this point, the amount of heat that can be converted into acoustic power (and vice versa) depends upon certain aspects of the stack (i.e. material properties, stack dimensions and the position of the stack in the tube).

Table 1. Physical properties of helium (He)

\begin{tabular}{|c|c|c|}
\hline \multicolumn{2}{|r|}{ Physical property } & Value \\
\hline \multirow{6}{*}{ 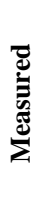 } & Density: $\mathrm{p}\left(\mathrm{kg} / \mathrm{m}^{2}\right)$ & 0.1741 \\
\hline & Isobaric specific heat :Cp $(\mathrm{kJ} / \mathrm{kg}, \mathrm{K})$ & 5.193 \\
\hline & Thermal conductivity: $\mathbb{K}(\mathrm{W} / \mathrm{m} \cdot \mathrm{K})$ & 0.145 \\
\hline & Specific heat ratio: $\mathbb{Y}$ & 1.667 \\
\hline & Specific ideal gas constant: $\mathbb{R}(\mathrm{kJ} / \mathrm{kg}, \mathrm{K})$ & 2.0771 \\
\hline & Dynamic viscosity: $\mu(\mathrm{kg} / \mathrm{m}, \mathrm{s})$ & $1.96 \times 10^{-5}$ \\
\hline \multirow{4}{*}{ 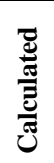 } & Sound of speed: $\mathrm{a}(\mathrm{m} / \mathrm{s}]$ & 985 \\
\hline & Thermal penetration depth: $\delta_{k}(\mathrm{~m}]$ & $3.819 \times 10^{-4}$ \\
\hline & Viscous penetration depth: $\mathbb{E}_{w}(\mathrm{~m})$ & $3.2 \times 10^{-4}$ \\
\hline & Prandtl number: $\sigma$ & 0.68 \\
\hline
\end{tabular}

We chose the plastic material for our stack design in that it: i) permits a low thermal conductivity in the $\mathrm{x}$ direction from the working gas, which it minimizes loss through conduction from hot to cold; ii) provides a greater heat capacity than the working gas because the capacity is less affected by the temperature oscillations of the nearby gas; iii) maintains a steady temperature gradient along the stack walls which increases the effectiveness of the gas in transporting thermal energy from the cold to the hot end of the stack. The dimensions of the stack (i.e. length, thickness, cross-sectional area and spacing between plates also influences the stack efficiency. This factor is considered important because of its effect on the temperature gradient, and because it used to determine how much the sound waves are perturbed and to determine the heat capacity and conductivity. The spacing between the plates in the stack is crucial in a properly functioning device. If the spacing between the plates is too narrow, the good thermal contact between the gas and the stack keeps the gas at a temperature similar to the stack. If the spacing between the plates is too wide, much of the gas is prevented from making good thermal contact with the stack which in turn does not transfer heat effectively to and from the stack. When the temperature difference across the stack is large enough, however, the air in the tube oscillates spontaneously. The stack design is also constrained by the requirement that the layers must be a few thermal penetration depths apart, with four thermal penetration depths being the optimum layer of separation. The addition of more plates to the stack increases the thermal exchange area, leading to an increased amount of heat flux and thus an increased overall efficiency of the device [10-11, 16-18]. Finally, the pores of the stack should be as small as possible in that the pore size is dependent upon the 
thermal penetration depth. Therefore, the pores should be designed so that the working gas and stack walls can transfer heat as effectively as possible, to avoid the creation of a larger surface area where turbulence occurs from losses that then disrupts the acoustic field $[6,20]$.

With a known frequency of operation, dimensionless heat and work flow equations were used to calculate and plot performance curves for various stack lengths and positions relative to the speaker. These equations were derived from the exact partial differential equations by making some simplifying assumptions [2, 6, 21]. The dimensionless forms of these equations, as derived by Tijani et al. [2] were obtained by using dimensionless parameters, as shown in Table 2. The equations for heat and work flows in a thermoacoustic element are:

Table 2. Normalized parameters used in dimensionless work and heat equations [2]

\begin{tabular}{|c|c|}
\hline & Normalized parameters \\
\hline \multirow{4}{*}{ 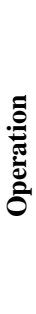 } & Drive ratio: $\mathrm{DR}=\frac{\mathbb{R}_{\mathrm{n}}}{\mathrm{P}_{\mathrm{m}}}$ \\
\hline & Norm. cooling power: $\hat{Q}_{z n}=\frac{Q_{s}}{D_{m}=\mathrm{A}}$ \\
\hline & Norm. acoustic power: $\tilde{W}_{\mathrm{n}}=\frac{w}{D_{m}=\mathrm{H}}$ \\
\hline & Norm. temperature difference: $\Delta \mathrm{T}_{\mathrm{mn}}=\frac{\Delta \mathrm{T}_{\mathrm{m}}}{\mathrm{T}_{\mathrm{m}}}$ \\
\hline है & Norm. thermal penetration depth: $\varepsilon_{k n}=\frac{E_{k}}{w_{n}}$ \\
\hline \multirow{3}{*}{ 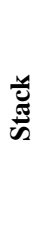 } & Norm. stack length: $\mathbb{L}_{2 \mathrm{~m}}=\mathrm{kL}_{\mathrm{s}}$ \\
\hline & Norm. stack center position: $\mathrm{x}_{2 \mathrm{n}}=\mathrm{kx} \mathrm{r}_{2}$ \\
\hline & Blockage ratio: $B=\frac{T_{0}^{g}}{\| m+2 ?^{2}}$ \\
\hline
\end{tabular}

$\mathrm{Q}_{\mathrm{cn}}=-\frac{\delta_{\mathrm{kn}} \mathrm{D}^{2} \sin \left(2 \mathrm{x}_{\mathrm{sn}}\right)}{8 \gamma(1+\sigma) \mathrm{A}}\left[\frac{\Delta \mathrm{T}_{\mathrm{mn}} \tan \mathrm{x}_{\mathrm{sn}}}{(\gamma-1) \mathrm{BL}_{\mathrm{sn}}} \frac{1+\sqrt{\sigma}+\sigma}{1+\sqrt{\sigma}}-(1+\sqrt{\sigma}\right.$

and

$W_{n}=\frac{\delta_{k n} L_{s n} D^{2}}{4 \gamma}\left[(\gamma-1) B \cos ^{2}\left(x_{s n}\right)\left\{\frac{\Delta T_{m n} \tan \left(x_{s n}\right)}{B L_{s n}(\gamma-1)(1+\sqrt[8]{\sigma}) \Lambda}-1\right.\right.$

where $\Lambda$ is used as intermediate variable and is defined as

$\Lambda=1-\delta_{\mathrm{kn}} \sqrt{\sigma}+\frac{1}{2} \sigma \delta^{2}{ }_{\mathrm{kn}}$

The axial thermal conduction in the stack was not considered and the axial heat transport was due to the thermoacoustic effects alone [2]. From the normalized of actual cooling power and acoustic power, the actual coefficient of performance, COP can be calculated using the normalized values. Therefore, the $\mathrm{COP}$ of a refrigeration system is expressed as
$\operatorname{cop}=\frac{Q_{\mathrm{cn}}}{W_{\mathrm{n}}}$

In Figures 3, 4 and 5 for the parameters and operation conditions were chosen up to this point in the design process in Table 3, the cooling power, acoustic power and coefficient of performance vs. stack center position for specify stack lengths are plotted respectively. These figures were used to determine the values of stack length $\left(\mathrm{L}_{\mathrm{g}}\right)$ and stack center position $\left(\mathrm{x}_{\mathrm{g}}\right)$ which is measured from the speaker face by maximize normalized cooling power and COP and minimize normalized required acoustic power and take the major consideration of the difficulty of the machinability of the stack especially of design channels which is generally very small and difficult to create without fracturing the material, so the stack length was chosen to be $\mathrm{L}_{\mathrm{g}}=0.07 \mathrm{~m}$. All the dimensions of the parallel stack were summarized in Table 4

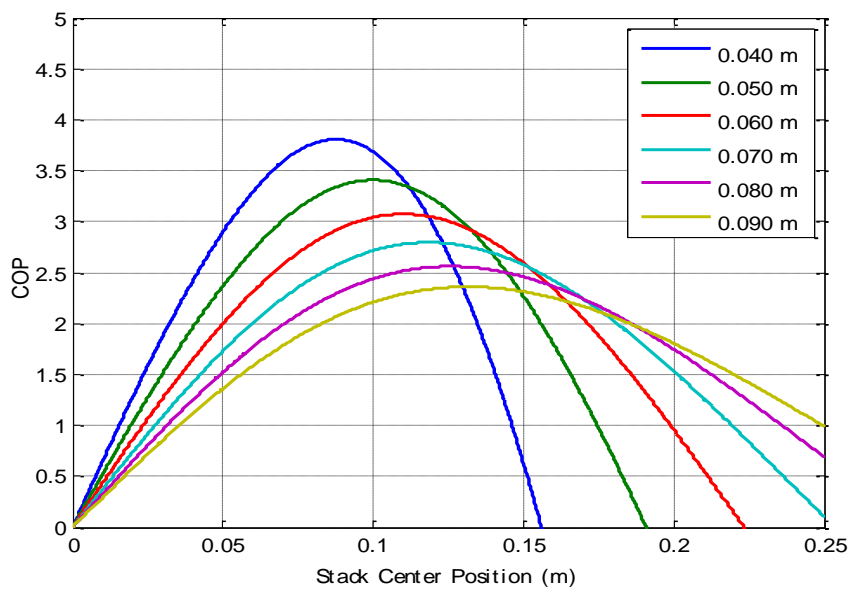

Fig. 3. Coefficient of performance vs. stack center position for various stack lengths.

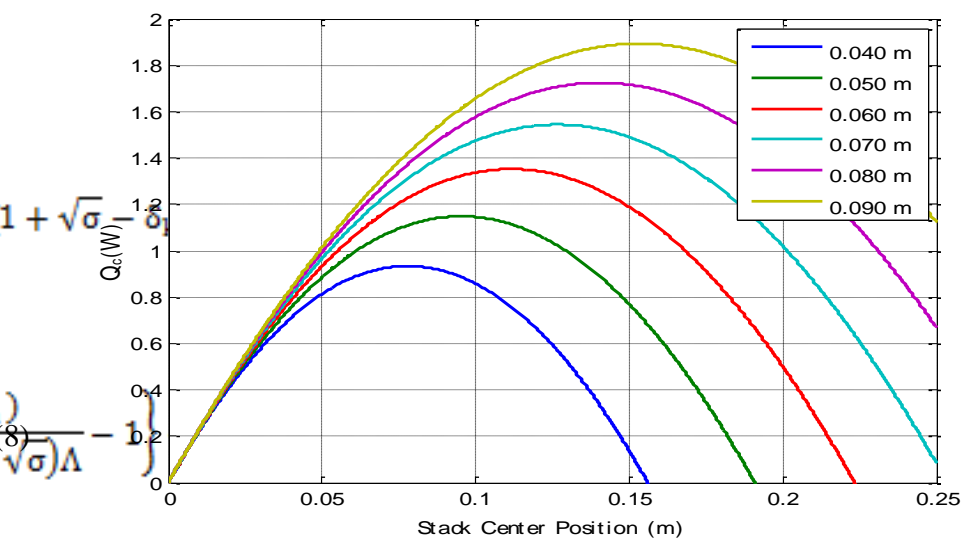

Fig. 4. Cooling power vs. stack center position for various stack lengths.

Table 3. Thermoacoustic refrigeration operating conditions.

\begin{tabular}{|l|c|}
\hline \multicolumn{1}{|c|}{ Parameters } & Value \\
\hline $\mathrm{P}_{\mathrm{m}}:$ Operating pressure $(\mathrm{kPa})$ & 101.325 \\
\hline $\mathrm{T}_{\mathrm{m}}:$ Mean temperature $(\mathrm{K})$ & 280.15 \\
\hline$\Delta \mathrm{T}:$ Desired temperature difference $(\mathrm{K})$ & 30 \\
\hline$f:$ Resonant frequency $(\mathrm{Hz})$ & 350 \\
\hline $\mathrm{DR}:$ Drive ratio & 0.01 \\
\hline
\end{tabular}




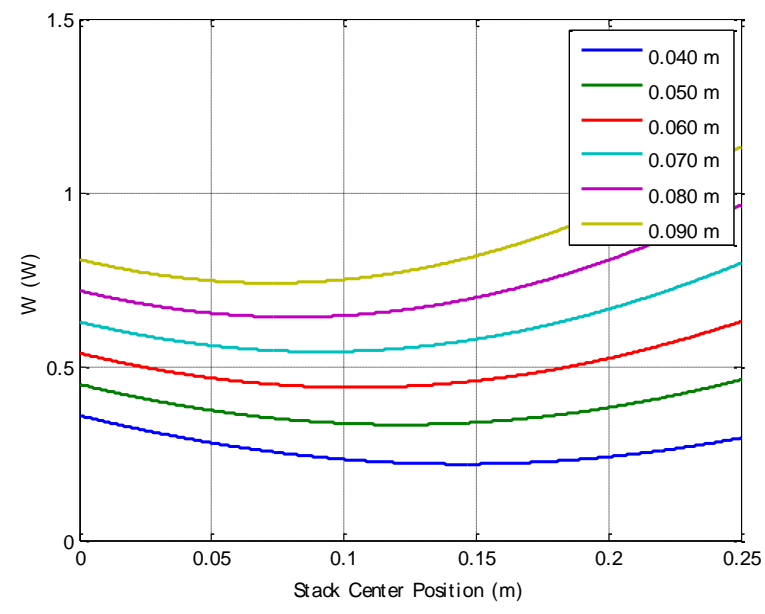

Fig. 5. Acoustic power vs. stack position for various stack lengths.

Table 4. Stack parameters

\begin{tabular}{|l|c|}
\hline \multicolumn{1}{|c|}{ Parameters } & Dimension \\
\hline $\mathrm{L}_{\mathrm{a}}:$ Stack length $(\mathrm{mm})$ & 70 \\
\hline $\mathrm{R}_{\mathrm{a}}:$ Stack center position $(\mathrm{mm})$ & 113 \\
\hline $2 \mathrm{I}_{\mathrm{g}}:$ Stack plate thickness $(\mathrm{mm})$ & 0.2 \\
\hline $2 \mathrm{y}_{\mathrm{g}}:$ Stack plate spacing $(\mathrm{mm})$ & 1.1 \\
\hline BR: Blockage ratio & 0.7160 \\
\hline
\end{tabular}

\subsection{Resonator Design}

The resonator is the body of the thermoacoustic refrigerator in which the sound waves propagate. It is used to store acoustic energy with minimize acoustic dissipation; obtain the proper phasing of the pressure and velocity, and to provide housing for the entire configuration. The resonator is composed of a large-diameter tube which contains the stack, a small-diameter tube, and a buffer volume. A contraction is used to connect the large and small diameter tubes.

We considered seven design facets when constructing our resonator. First, we ensured that the cross-sectional area of a resonance tube was circular to minimize the inner circumference of the tube for a given cross-sectional area and the total dissipation at the surface. This was expressed equation (11)

$$
\frac{\mathrm{dW} W_{\text {loss }}}{\mathrm{dS}}=\frac{1}{4} \rho_{\mathrm{m}}\left|\mathrm{u}_{1}\right|^{2} \delta_{v} \omega+\frac{1}{4} \frac{\left|\mathrm{P}_{1}\right|^{2}}{\rho_{\mathrm{m}} \mathrm{a}^{2}}(\gamma-1) \delta_{\mathrm{k}} \omega
$$

where is $\mathrm{u}_{1}$ is the particle velocity. The first term on the righthand side is the kinetic energy dissipated by viscous shear, and the second is the energy dissipated by thermal relaxation in the boundary layer. As the total of these two loss mechanisms is proportional to the surface area of the resonator, by reducing the surface area we increase the efficiency. Our second consideration required that the length of the resonator be a quarter that of the wavelength $\lambda(\lambda=2 . \mathrm{L})$. A quarterwavelength resonator will dissipate only half the energy dissipated by the half-wavelength resonator. Next, we ensured that the resonator have a large diameter section to provide our stack with rigidity, and very low thermal conductivity or insulation, to prevent heat loss during thermoacoustic heat pumping. We also designed our resonator with walls that were sufficiently thick enough to operate under high pressure, in which several discrete parts were assembled to ensure its safe operation. In our fifth design consideration, we wanted our small diameter section to be approximately half of that of the large diameter section, and have sufficient structural integrity to prevent any pressure or sound loss. We used copper to construct all components of our resonator except the stack housing, which was made of a material with low thermal conductivity. We tapered the connection between the different sized tubes in our resonator to avoid a dissipation of power and designed the final product to be compact, light, and robust enough for propagating sound waves. All of the requisite characteristics for our resonator design are provided in Table 5 $[2,6,21-23]$.

Table 5. General characteristic of resonator parameters

\begin{tabular}{|l|c|}
\hline \multicolumn{1}{|c|}{ Parameters } & Value \\
\hline $\mathrm{D}_{1}:$ Diameter of large tube $(\mathrm{mm})$ & 50 \\
\hline $\mathrm{L}_{1}$ Length of the large diameter $(\mathrm{mm})$ & 170 \\
\hline $\mathrm{D}_{2}:$ Diameter of small tube $(\mathrm{mm})$ & 25 \\
\hline $\mathrm{L}_{2}:$ Length of the small diameter $(\mathrm{mm})$ & 300 \\
\hline $\mathrm{D}_{2} / \mathrm{D}_{1}:$ ratio between small diameter and large diameter & 0.5 \\
\hline $\mathrm{L}_{\text {tot }}:$ Total length of resonator $(\mathrm{mm})$ & 470 \\
\hline
\end{tabular}

\subsection{Heat Exchanger Design}

The heat exchanger, which functions as a heat pump to transfer heat either into or out of a system, is driven by the acoustics produced from the stack and is attached to both ends of the stack. In order to achieve the optimum performance in our exchanger, we ensured that the axial length of the hot-side heat exchanger have twice the acoustic displacement amplitude of the gas as shown in equation 12. This displacement amplitude is expressed as

$\mathrm{L}_{\mathrm{hx}}=2 . \xi=2 . \frac{\mathrm{u}_{1}}{\omega}$

Where $u_{1}$ is the $\mathrm{x}$-component of the velocity of the longitudinal wave. When a heat exchanger is too long, some of fluid parcels only make contact with the ends of the heat exchanger; when it is too short, some parcels jump past the heat exchanger. In order to prevent the total blockage of any gaps in the stack by a heat exchanger strip, we ensured that the heat exchanger strips and the nearby stack plates are nonparallel to each other [20, 24-25].

We used the same heat exchanger design developed by Nsofor et al. in which the inside and outside diameters of the exchanger tube are $59 \mathrm{~mm}$ and $65 \mathrm{~mm}$ respectively [25]. The width of the copper fins $(0.15 \mathrm{~mm}$ thick) in the direction of the oscillating gas was $6.6 \mathrm{~mm}$ and the gap between the fins was $0.95 \mathrm{~mm}$

\subsection{Acoustic Driver}

The purpose of the acoustic driver is to provide the total acoustic power used by the stack to transfer heat and dissipate that heat within the different components of the refrigerator. A higher driver performance means that we can obtain the necessary acoustic power without using high electrical currents that can damage the coil. Consequently, because the power is obtained without coil damage, the performance of the entire refrigerator is also increased [2, 26-27]. We used legacy LS328 3.5-Inch 120 Watt two way speakers in our acoustic driver. 


\section{Measurements}

After successfully constructing our thermoacoustic refrigeration device, we tested it to optimize the optimum frequency at 90 minute intervals, and recorded the temperatures of the upper and lower thermocouple in the heat exchanger. During these 90 minute runs, we found that the optimum frequency for refrigerators was $350 \mathrm{~Hz}$.

\subsection{Stack Temperature Variation}

The variation in the temperature-time history above and below the stack during our 90 minute testing runs at a frequency of $350 \mathrm{~Hz}$ is displayed in Figure 6. The top red line shows the readings of the warm thermocouple in the hot heat exchanger, while the bottom blue line shows the readings of the cooler thermocouple cold heat exchanger. As the time passes, the temperature discrepancy between the hot end and cold end of the resonator tube increased for the time $t=70 \mathrm{~min}$ then reached a steady state. The rate of change of the temperature difference began to decrease, however, with the hotter end transferring heat back to the cooler, which in turn resulted in an increase in the temperature of the bottom end of the stack.

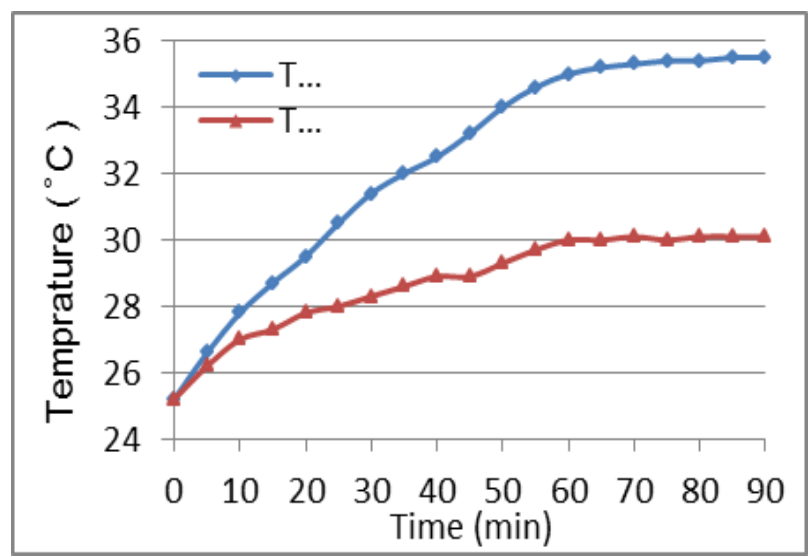

Fig. 6. Temperature -Time history of above and below the stack during a period of time at frequency of $350 \mathrm{~Hz}$.

\subsection{Simulation}

We used the commercial CFD package "SolidWorks" to simulate the performance of the thermoacoustic refrigerator using the data collected during our experimental measurements. This program was most efficient and accurate in describing the temperature and velocity within the refrigerator as shown in Figure 7.

\section{Conclusion}

We elucidated in great detail our optimal design strategy using the different components of our standing wave thermoacoustic refrigerator. We conducted these experimental investigations to study the effect of stack temperature variation, stack length and stack position on the coefficient of refrigeration performance. We also used our CFD to simulate the performance of thermoacoustic refrigerator, particularly the temperature and velocity trajectories within the refrigerator. Though our experimental and simulation results were reasonably good, quantitative differences were due to losses that were unaccounted in the present theoretical analysis.

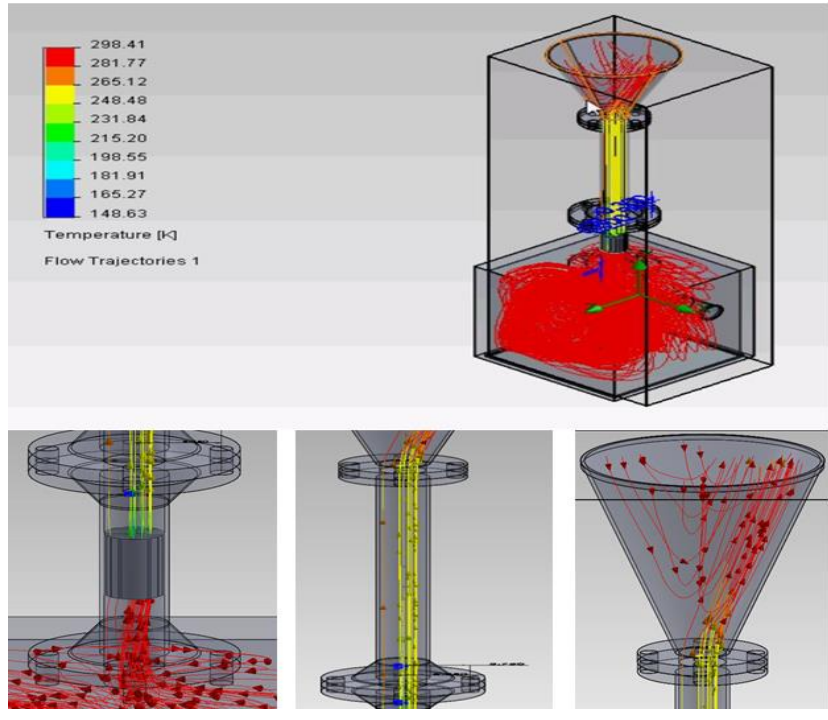

Fig. 7. Represents the movement of helium atoms in the thermoacoustic refrigerator.

\section{References}

[1] SL. Garrett, Thermoacoustic engines and refrigerators, Am J Phys 72 (1) (2004): 11-17. http://dx.doi.org/10.1119/1.1621034

[2] M. Tijani, J. Zeegers, A. Waele, Design of thermoacoustic refrigerators, Cryogenics 42 (1) (2002): 49-57. http://dx.doi.org/10.1016/S0011-2275(01)00179-5

[3] GW. Swift, Thermoacoustics - a unifying perspective for some engines and refrigerators, Melville, NY: Acoustical Society of America; 2002.

[4] M. Akhavanbazaz, M. Siddiqui, R. Bhat, The impact of gas blockage on the performance of a thermoacoustic refrigerator, Experimental Thermal and Fluid Science 32 (1) (2007): 231-239. http://dx.doi.org/10.1016/j.expthermflusci.2007.03.009

[5] P. Ceperley, A pistonless Stirling engine - the travelling wave heat engine, J Acoust Soc Am 66 (5) (1979): 15081513. http://dx.doi.org/10.1121/1.383505

[6] T. Ryan, Design and control of a standing-wave thermoacoustic refrigerator, Master thesis, Swanson School of Engineering, University of Pittsburgh, 2009.

[7] P. Andersson, Review of thermoacoustic refrigeration; description of a simple thermoacoustic refrigerator for demonstration purpose. Master Thesis, Department of Applied Acoustics, Chalmers University of Technology. Göteborg, 1999.

[8] TJ. Hofler, Thermoacoustic refrigerator design and performance, Ph.D. Dissertation, Physics Department, University of California, San Diego, 1986.

[9] M. Wetzel, C. Herman, Design optimization of thermoacoustic refrigerators. Int J Refrig 20 (1) (1997): 321. 
http://dx.doi.org/10.1016/S0140-7007(96)00064-3

[10] H. Babaei, K. Siddiqui, Design and optimization of thermoacoustic devices, Energy Conversion and Management 49 (12) (2008): 3585-3598. http://dx.doi.org/10.1016/j.enconman.2008.07.002

[11] EC. Nsofor, A. Ali, Experimental study on the performance of the thermoacoustic refrigerating system, Applied Thermal Engineering 29 (13) (2009): 2672-2679. http://dx.doi.org/10.1016/j.applthermaleng.2008.12.036

[12] D. Russell, P. Weibull, Tabletop thermoacoustic refrigerator for demonstrations, American Journal of Physics 70 (12) (2002): 1231-1233.

http://dx.doi.org/10.1119/1.1485720

[13] M. Tijani, J. Zeegers, A. Waele, Prandtl number and thermoacoustic refrigerators, J Acoust Soc Am 112 (1) (2002): 134-143. http://dx.doi.org/10.1121/1.1489451

[14] A. Campo, M. Papari, E. Abu-Nada, Estimation of the minimum Prandtl number for binary gas mixtures formed with light helium and certain heavier gases: Application to thermoacoustic refrigerators, Applied Thermal Engineering 31(16) (2011): 3142-3146. http://dx.doi.org/10.1016/j.applthermaleng.2011.05.002

[15] GW. Swift, Thermoacoustic engines and refrigerators, Physics Today 48 (7) (1995): 22-28. http://dx.doi.org/10.1063/1.881466

[16] B.G. Prashantha, M.S. Govinde Gowdab, S. Seetharamu, Effect of Mean Operating Pressure on the Performance of Stack-Based Thermoacoustic Refrigerator, Int. J. of Thermal \& Environmental Engineering Volume 5 (1) (2013): 83-89. http://dx.doi.org/10.5383/ijtee.05.01.009

[17] Q. Tu, Q. Li, F. Guo, J. Wu, J. Liu, Temperature difference generated in thermo-driven thermoacoustic refrigerator. Cryogenics 43 (9) (2003): 515-522. http://dx.doi.org/10.1016/S0011-2275(03)00131-0
[18] W. Slaton, J. Zeegers, Thermoelectric power generation in a thermoacoustic refrigerator, Applied Acoustics 67 (5) (2006) 450-460.

http://dx.doi.org/10.1016/j.apacoust.2005.06.008

[19] L. Zoontjens, Numerical investigations of the performance and effectiveness of thermoacoustic couples, Ph.D. Thesis, University of Adelaide, School of Mechanical Engineering, 2008.

[20] JC. Wheatly, TJ. Hofler, GW. Swift, A. Migliori, Understanding some simple phenomena in thermoacoustics with applications to acoustical heat engines, Am J Phys 53 (2) (1985): 147- 162. http://dx.doi.org/10.1119/1.14100

[21] GW. Swift, Thermoacoustic engines. J Acoust Soc Am 84 (4) (1988): 1146-1180. http://dx.doi.org/10.1121/1.396617

[22] Bheemsha, N. Ramesh, G. Pundarika, Design of the Resonator Tube and Buffer Volume for Thermo Acoustic Refrigerator, International journal of Advanced Scientific and Technical Research 2 (1) (2011): 276-288.

[23] I. Paek, J. Braun, L. Mongeau, Evaluation of standingwave thermoacoustic cycles for cooling applications, International Journal of Refrigeration 30 (6) (2007): 1059-1071.

http://dx.doi.org/10.1016/j.ijrefrig.2006.12.014

[24] M. Tijani, S. Spoelstra, Study of a coaxial thermoacousticStirling cooler, Cryogenics 48 (1-2) (2008): 77-82. http://dx.doi.org/10.1016/j.cryogenics.2008.01.001

[25] E. Nsofor, S. Celik, X. Wang, Experimental study on the heat transfer at the heat exchanger of the thermoacoustic refrigerating system, Applied Thermal Engineering 27 (14-15) (2007): 2435-2442. http://dx.doi.org/10.1016/j.applthermaleng.2007.03.008

[26] L. Zoontjens, C. Howard, A. Zander, B. Cazzolato, Development of a low-cost loudspeaker-driven thermoacoustic refrigerator, Proceedings of ACOUSTICS 2005 .

[27] Q. Tu, V. Gusev, M. Bruneau, C. Zhang, L. Zhao, F. Guo, Experimental and theoretical investigation on frequency characteristic of loudspeaker-driven thermoacoustic refrigerator, Cryogenics 45 (12) (2006): 739-746. http://dx.doi.org/10.1016/j.cryogenics.2005.09.004 